\title{
Investigando Evidências de Validade de Conteúdo e Estrutural em Tarefas de um Treino Cognitivo para Idosos ${ }^{1}$
}

\author{
Mariana Teles Santos Golino² \\ Universidade Federal da Bahia, Vitória da Conquista-BA, Brasil \\ Patrícia Waltz Schelini \\ Universidade Federal de São Carlos, São Carlos-SP, Brasil \\ Hudson Fernandes Golino ${ }^{3}$ \\ Universidade Salgado Oliveira, Niterói-RJ, Brasil \\ Bruna Luísa de Souza Pereira, Lucas Matias Felix \\ Universidade Federal da Bahia, Vitória da Conquista-BA, Brasil
}

RESUMO

O presente artigo objetiva reportar os resultados de dois estudos de validade conduzidos em um treino cognitivo para idosos. O modelo teórico adotado para a intervenção previu a estimulação de cinco habilidades cognitivas. Um estudo de validade de conteúdo foi conduzido por meio de uma análise de juízes com um grupo de 16 especialistas na área. Os resultados apontaram para uma baixa concordância entre os avaliadores (índice Fleiss Kappa =0,11; $-0,02<$ icc $<0,12, p=0,18$ ). Um estudo de validade estrutural investigou a replicabilidade do modelo teórico em uma análise fatorial bayesiana. Os resultados apontaram para adequação e convergência do modelo de Cinco Fatores (teste Diagnóstico de Heidel: $p<0,05$ para os itens), porém, a distribuição das tarefas não foi integralmente confirmada no modelo empírico. Conclui-se pela confirmação parcial do modelo teórico adotado para o treino.

Palavras-chave: treino cognitivo, validade de conteúdo, validade estrutural.

ABSTRACT - Investigating evidence of content and structural validity in cognitive training tasks for the elderly The present article reports the results of two validity studies conducted in a cognitive training session for the elderly. The theoretical model adopted for the intervention predicted the stimulation of five cognitive abilities. A content validity study was conducted through a panel of judges including 16 experts in the field. The results pointed to a low agreement between the evaluators (Fleiss Kappa index $=0.11 ;-0.02<\mathrm{icc}<0.12, p=0.18)$. A structural validity study investigated the replicability of the theoretical model in a Bayesian factor analysis. The results pointed to fit and convergence of the five-factor model (Heidel's diagnostic test: $p<0.05$ for the items); however, the task distribution was not fully confirmed in the empirical model. The report concludes with partial confirmation of the theoretical model adopted for the training.

Keywords: Cognitive training, Content validity, Structural validity.

RESUMEN - Investigando evidencias de validez de contenido y estructural en tareas de un entrenamiento cognitivo para ancianos

Este artículo tuvo como objetivo informar los resultados de dos estudios de validez realizados en un entrenamiento cognitivo para ancianos. El modelo teórico adoptado para la intervención previó la estimulación de cinco habilidades cognitivas. Se realizó un estudio de validez de contenido que fue conducido por medio de un análisis de jueces con un grupo de 16 especialistas en el área. Los resultados mostraron baja concordancia entre los evaluadores (índice FleissKappa $=0,11 ;-0,02<\mathrm{icc}<0,12, p=0,18$ ). Un estudio de validez estructural investigó la replicabilidad del modelo teórico en un análisis factorial bayesiano. Los resultados apuntaron para adecuación y convergencia del modelo de cinco factores (test diagnóstico de Heidel: $p<0,05$ para los ítems), sin embargo, la distribución de tareas no fue integralmente confirmada en el modelo empírico. Los resultados confirmaron parcialmente el modelo teórico adoptado para el entrenamiento.

Palabras clave: entrenamiento cognitivo, validez de contenido, validez estructural.

\section{Introdução}

A investigação do impacto de intervenções cognitivas sobre o desempenho mental em idosos tem sido foco de interesse crescente em Psicologia e Neurociência na última década (Hertzog, Kramer, Wilson, \& Lindenberger, 2009; Chein \& Morrison, 2010; Karbach \& Verhaeghen, 2014; Melby-Lervag \& Hulme, 2013). Os

${ }^{1}$ Agradecimentos: Fundação de Amparo à Pesquisa do Estado da Bahia (FAPESB)

2 Endereço para correspondência: Universidade Federal da Bahia, Instituto Multidisciplinar em Saúde. Rua Rio de Contas, 58, Gabinete 24, 45029-094, Vitória da Conquista-BA. Tel.: (77) 98119-9702. E-mail: marianatsantos84@gmail.com

${ }^{3}$ Endereço para correspondência: Department of Psychology, University of Virginia, USA. 485, McCormick Road, Gilmer Hall, Room 102 , Charlottesville, VA 22903. E-mail: hfg9s@virginia.edu 
resultados desses estudos, em grande medida, sugerem que intervenções adequadas produzem amplos efeitos de aprendizagem, aumento no desempenho das habilidades treinadas e, mais raramente reportados, efeitos de generalização para atividades cotidianas ou habilidades cognitivas não treinadas (Bergman-Nutley et al., 2011; Jaeggi, Buschkuehl, Jonides, \& Perrig, 2008; Klingberg, 2010; Strobach, Frensch, \& Schubert, 2012).

Os programas de intervenção, nos quais mecanismos cognitivos específicos são isolados e treinados, também consistem em uma maneira direta e experimentalmente controlada de investigar o grau de plasticidade no funcionamento intelectual e vêm sendo cada vez mais utilizados como uma metodologia de reabilitação, pois podem produzir um impacto positivo na prevenção ou amenização do declínio mental decorrente de quadros demenciais (Valenzuela \& Sachdev, 2009). A validade das intervenções é investigada experimentalmente com a verificação dos efeitos dos ganhos cognitivos alcançados após a intervenção (e atribuídos ao treino), que podem ser acessados por meio de medidas cognitivas pré e pós-treino (ganhos imediatos) ou por meio de acompanhamentos a longo prazo (follow-up) para verificação da durabilidade e generalização dos ganhos (Kelly et al., 2014; Martin, Clare, Altsgassen, Cameron, \& Zehnder, 2011)

Os primeiros trabalhos com uso de delineamento experimental para verificar o impacto de intervenções padronizadas datam da década de 1980 (Schaie \& Willis, 1986; Willis \& Nesselroade, 1990) e, desde então, as evidências acumuladas na área vêm apontando de forma consistente que: 1. É possível aumentar o desempenho em tarefas cognitivas que mensuram as habilidades cognitivas treinadas na intervenção - efeito near transfer: em estudo de meta-análise com ensaios clínicos randomizados, encontrou-se forte tamanho de efeito para os ganhos cognitivos em habilidades treinadas (WMD $=1,07$; $\mathrm{IC}=0,32-1,83 ; z=2,78 ; p=0,006, N=3,194)$ (Valenzuela \& Sachdev, 2009); e 2) Variáveis sociodemográficas, clínicas e diferenças individuais são fatores preditivos para o impacto das intervenções sobre o funcionamento mental, com destaque para o nível de escolaridade, a prática de exercícios físicos, preservação da saúde mental e o suporte social (ver revisão de Hertzog et al., 2009).

Porém, outras questões ainda permanecem em aberto e não alcançaram consenso entre os pesquisadores: 1. Efeitos de transferência do ganho do treino para domínios cognitivos distantes das habilidades treinadas - far transfer - são escassamente reportados, porém, em revisão sistemática de 31 estudos conduzida por Kelly et al. (2014), identificou-se que sessões de treino adaptativas e períodos mais longos de intervenção produziram melhores resultados para far transfer; 2. Generalização das aprendizagens obtidas durante a intervenção para resolução de problemas cotidianos; e 3. Durabilidade temporal dos efeitos do treino, ambos escassamente investigados devido ao reduzido número de estudos de follow-up e, portanto, com poucas evidências a seu favor. (ver revisões sistemáticas: Law, Barnett, Yau, \& Gray, 2014; Reijinders, Van Heugten, \& Van Boxtel, 2013; Tardirf \& Smard, 2011. Ver também estudos de meta-análise: Papp, Stephen, \& Peter, 2009; Martin et al., 2011). Uma exceção importante de evidência de generalidade e durabilidade pode ser observada no projeto Advanced Cognitive Training for Independent and Vital Elderly (ACTIVE), em que o estudo de follow-up de 10 anos reportou menor dificuldade do grupo de treino com atividades instrumentais da vida diária (generalização): (a) Grupo de Memória: tamanho de efeito=0,48, IC $99 \%=0,12-0,84$; (b) Grupo de Raciocínio: tamanho de efeito $=0,38$, IC $99 \%=0,02-0,74 ; 3$; (c) Grupo de Velocidade de Processamento: tamanho do efeito $=0,36$, IC $99 \%=0,01-0,72$. Foram reportados, também, efeitos de durabilidade para Raciocínio (tamanho do efeito $=0,23$, IC $99 \%=0,09-0,38$ ) e Velocidade de Processamento (tamanho do efeito $=0,66$, IC 99\%=0,43-0,88) (Rebok et., al., 2014).

Tais pontos de divergência seguem dividindo a área no tocante à possibilidade de mudanças cognitivas estruturais em um construto tão estável como a cognição. Em recente revisão dos aspectos metodológicos da área, Shubert, Strobach e Karbach (2014) argumentam que o campo de investigações sobre intervenções do tipo treino cognitivo ainda está superando a sua infância. Os autores apontam algo já notório nas divulgações dos resultados da área: durante muitas décadas houve um predomínio de estimativas entusiastas, exageradas e com viés de confirmação sobre os efeitos dos treinos. Alguns autores argumentam que, durante a análise dos resultados reportados, o posicionamento dos pesquisadores quase sempre extrapola os limites do que é possível inferir com os dados de que dispõem (Hertzgov et al., 2009; Salthouse, 2006).

Esse movimento de crédito desproporcional sobre as evidências disponíveis na área tem favorecido ou contribuído, mesmo que indiretamente, para o lançamento e venda de produtos, como games ou websites, que prometem aumentar o funcionamento cognitivo e o desempenho mental da população. É possível observar que muitos programas de intervenção estão sendo transformados em produtos comerciais, como o Brain Age (Nintendo), que promete melhorar o funcionamento cognitivo durante o envelhecimento. Porém, para a maioria desses softwares comerciais faltam evidências que amparem suas promessas (para uma descrição de tais produtos, acessar: http://www.sharpbrains.com). O fato de termos um movimento econômico e mercadológico paralelo à produção científica na área da intervenção cognitiva demanda que os pesquisadores redobrem seus esforços no sentido de tecer conclusões cuidadosas sobre seus achados.

O problema das inferências superestimadas dos resultados em favor da eficácia das intervenções, que 
caminha em paralelo com as promessas dos produtos comerciais, está intimamente entrelaçado com uma outra questão bastante delicada, porém pouco discutida na literatura: em geral, os estudos divulgam intervenções por meio da simples identificação das habilidades treinadas (como, por exemplo: treino de memória episódica, treino de funções executivas, treino de atenção e velocidade). Porém, lança-se a questão: como afirmar que o treino está, de fato, estimulando as habilidades divulgadas? As tarefas das intervenções são elaboradas de acordo com os modelos teóricos que amparam a perspectiva do estudo e do pesquisador, contudo, há uma escassez de estudos voltados para a confirmação e replicação empírica de tais modelos. Esse problema central compromete o processo inferencial dos efeitos de transferência do treino: como afirmar quais são as habilidades treinadas e as habilidades não treinadas durante a intervenção? Essa determinação é feita com base nos modelos teóricos e a partir da suposição dos pesquisadores de que as tarefas elaboradas, de fato, alcançam as habilidades pretendidas. No geral, não há uma preocupação em conduzir estudos para levantar evidências robustas de que as tarefas da intervenção estão adequadas para as habilidades-alvo, o que denuncia um problema central nos processos de construção e testagem das intervenções.

Em revisão da literatura internacional sobre treino cognitivo para idosos, analisou-se: 1 . as principais publicações de dois ensaios clínicos randomizados de grande porte - o SLS-Seattle Longitudinal Study (Schaie, Willis, \& Caskie, 2004; Schaie, 2005) e o ACTIVE - Advanced Cognitive Training for Independent and Vital Elderly Ball et al., 2002; Bahar-Fuchs et al., 2013; Jobe et al., 2001; Rebok et al, 2014); 2. dois estudos de revisão sistemática (Law, Barnett, Yau, \& Gray, 2014; Tardif \& Smard, 2011); e 3. quatro estudos de meta-análise (Reijinders, Van Heugten, \& Van Boxtel, 2013; Kelly et al., 2014; Martin et al., 2011; Valenzuela \& Sachdev, 2009). Nenhum dos estudos citados reportou a condução de estudos de validade para verificar a replicabilidade nos dados empíricos do modelo teórico adotado na determinação das habilidades cognitivas que foram alvo da intervenção.

O mesmo padrão de resultado pode ser observado na revisão da literatura nacional sobre treino cognitivo para idosos. Foram pesquisados, entre janeiro de $1990 \mathrm{e}$ julho de 2016, os termos (em português e inglês): "treino cognitivo", "estimulação cognitiva", "envelhecimento", "idoso" e "brasileiro" nas bases SciELO, PsycInfo e PubMed. Alguns trabalhos de tese e dissertação foram incluídos para acesso a uma especificação mais detalhada das pesquisas. Não foram identificados estudos de meta-análise, portanto, consultou-se os estudos experimentais individuais. Um total de 23 estudos nacionais foram analisados ${ }^{4}$. Nenhum deles reportou a condução de investigações sobre evidências de validade do programa que sustentem o modelo teórico adotado para definir as habilidades cognitivas treinadas. Conclui-se, assim, por uma escassez, no campo de investigações de intervenções cognitivas, de estudos de validade das tarefas dos treinos.

Como alternativa para avançar na investigação da validade das intervenções sustenta-se a incorporação, pela área, de métodos psicométricos para validação dos programas de treino cognitivo, mais especificamente, por meio da condução de investigação de fontes de evidência de validade das tarefas do treino. Dessa forma, é possível investigar a adequação das tarefas do treino às habilidades-alvo pretendidas e testar a replicabilidade do modelo teórico subjacente à intervenção. Nessa perspectiva, sugere-se que as tarefas do treino sejam entendidas como itens de um teste, na medida em que pretendem eliciar comportamentos (respostas) do indivíduo. Como agentes eliciadores dos comportamentos, as tarefas do treino devem ser capazes de ativar a capacidade do indivíduo de responder a elas (habilidade), juntamente com o grau de demanda ou esforço para sua resolução (dificuldade). Habilidade e dificuldade são o que os filósofos da ciência chamam de definições constitutivas, ou seja, são conceitos complementares que são definidos em termos de um e do outro ao mesmo tempo (Andrich, 2004). Para determinar a relação e adequação entre os agentes eliciadores (tarefas) e os comportamentos (habilidade) são conduzidas investigações de evidências de validade.

De acordo com Markus e Borsboom (2013), do ponto de vista epistemológico, é possível entender o estudo de validade em diferentes perspectivas: empiricismo descritivo, empiricismo explicativo, construtivismo realista e realismo teórico. Cada corte epistemológico impactou profundamente a construção dos estudos de validade no campo da mensuração. Para este estudo, será adotada a perspectiva empiricista explicativa, na qual se entende validade como o grau em que as evidências fornecem suporte para o processo inferencial dos resultados de um teste (validade de construto). Adicionalmente, as fontes de validade de um conjunto de tarefas podem ser investigadas a partir da associação dos escores com critérios externos ao teste (validade de critério), ao grau de representatividade ou adequação dos itens ao construto latente pretendido (validade de conteúdo), à estrutura encontrada na relação entre

${ }^{4}$ Aramaki \& Yassuda (2011); Brum (2012), Carvalho, Neri, \& Yassuda (2010); Chariglione (2010); Chariglione (2014); Dias \& Lima (2012); Irigaray, Filho, \& Schneider (2010); Irigaray, Schneider, \& Gomes (2004); Lasca (2003); Lima-Silva \& Yassuda (2010); Lima-Silva et al. (2010); Lima-Silva et al. (2011); Olchik (2008); Oliveira et al. (2014); Paulo \& Yassuda (2012); Santos (2010) ; Silva \& Yassuda (2009); Soares, Santana, \& Rabelo (2015); Tavares, Shimidt, \& Witter (2015); Teixeira-Fabrício et al. (2012); Wood (2000); Yassuda et al. (2006); Zimmerman et al. (2014). 
as tarefas e suas variáveis latentes (validade estrutural), dentre tantas outras fontes reportadas na literatura (Markus \& Borsboom, 2013).

O presente artigo tem como objetivo apresentar os resultados da condução de dois estudos de investigação de fontes de evidência de validade para um programa de treino cognitivo conduzido em idosos saudáveis. $\mathrm{O}$ primeiro estudo investigou evidências de validade de conteúdo por meio de uma análise de juízes, na qual um grupo de 16 especialistas na área julgaram a adequação das tarefas do treino para o alcance das habilidades pretendidas. O segundo estudo investigou as evidências de validade estrutural por meio da análise do desempenho dos participantes do grupo experimental nas tarefas do treino (e não nas medidas pré e pós-treino), buscando elucidar a estrutura fatorial subjacente às tarefas do programa. O programa de intervenção tinha um desenho tal que os participantes inicialmente respondiam às tarefas de forma a que o profissional conduzindo a intervenção pudesse verificar a sua capacidade basal, isto é, a sua capacidade de realizar as tarefas em diferentes domínios. Somente após esse momento inicial, a intervenção propriamente dita ocorria. Dessa forma, no segundo estudo, os dados analisados são oriundos dessa primeira etapa do programa, de forma que o efeito da intervenção no desempenho dos indivíduos é minimizado.

É importante destacar que, nas etapas de construção do protocolo de intervenção, adotou-se um modelo teórico de cinco domínios cognitivos supostamente treinados por tarefas distintas. Ressalta-se que os autores se fundamentaram nos conceitos da Psicologia Cognitiva para definição e compreensão das habilidades cognitivas treinadas. A escolha de tal estrutura foi norteada por quatro conjuntos de evidências reportadas na literatura: 1. Treino de memória episódica: um dos domínios mais investigados na área, com estudos de revisão sistemática comumente reportando efeitos significativos de ganho de treino, assim como são observados tamanhos de efeito fortes (cerca de 0,7) em medidas objetivas reportados em estudos de meta-análises (Belleville et.al., 2006; Verhaeghen, Marcoen, \& Goossens, 1992); 2. Intervenções do tipo multidomínio, com grande diversidade de tarefas, comumente apresentam melhores resultados quando comparadas com treinos exclusivos de uma habilidade (Auffray \& Juhel, 2001; Willis et. al., 2006); 3. Optou-se por incluir exercícios de atenção e velocidade anteriores ao treino de memória, com base em achados que apontam um melhor aproveitamento do treino de memória neste formato (pretraining), potencializando o tamanho de efeito (de intervalos que variaram de $0,10-1,21$ para intervalos de 0,88-1,18 (Gates et. al., 2011). 4. Inclusão de tarefas de Memória de Trabalho: em revisão sistemática, Hertzgov et. al. (2008) reportaram que os estudos que incluíram, além de técnicas de memorização, tarefas de memória de trabalho, apresentaram melhores resultados em generalização dos ganhos.

\section{Método}

\section{Estudo 1: Validade de Conteúdo}

O estudo de validade de conteúdo das tarefas do treino teve como objetivo conduzir uma análise de juízes para verificar o grau de concordância em relação à adequação das tarefas do treino às habilidades cognitivas pretendidas.

\section{Participantes}

Grupo de 16 especialistas na área da Psicologia Cognitiva, Avaliação Psicológica e áreas afins, sendo 14 doutores e 2 mestres.

\section{Instrumentos}

O treino cognitivo consistiu em 115 itens distribuídos em 13 tarefas. Ressalta-se que a mesma quantidade de itens precedeu o treino propriamente, consistindo em itens de teste para familiarização do sujeito às tarefas e averiguação de seu nível de habilidade. Cada tarefa pretendeu estimular um domínio cognitivo específico, como apresentado na Tabela 1.

Tabela 1

Tarefas e Domínios Cognitivos Pretendidos

\begin{tabular}{lcl}
\hline \multicolumn{1}{c}{ Domínio Cognitivo } & Tarefa (número de itens) & Descrição \\
\hline Atenção concentrada & Labirintos (9) & Encontrar o caminho que liga o início ao final de um \\
e velocidade de & & labirinto, sem ultrapassar as linhas, o mais rápido que \\
processamento & puder. Em seguida, alguns labirintos são repetidos com \\
& & tempo estipulado de execução (metade do tempo gasto \\
& & na primeira execução).
\end{tabular}

Busca Visual (9)

Identificar com um traço estímulos-alvo dentro de um conjunto de estímulos distratores, o mais rápido que puder. Em seguida, alguns itens são repetidos com tempo estipulado de execução (metade do tempo gasto na primeira execução).

Estimulação sonora (10) Identificar, em um conjunto de palavras, aquela que está errada ou não existe. 
Tabela 1 (continuação)

Tarefas e Domínios Cognitivos Pretendidos

\begin{tabular}{lll}
\hline \multicolumn{1}{c}{ Domínio Cognitivo } & Tarefa (número de itens) & \multicolumn{1}{c}{ Descrição } \\
\hline $\begin{array}{l}\text { Habilidades } \\
\text { perceptomotoras }\end{array}$ & Cópias (6) & Reproduzir figuras com e sem a exposição delas. \\
\hline Memória visual & Visualizando figuras (11) & $\begin{array}{l}\text { Visualizar uma figura e, em seguida, responder a perguntas } \\
\text { sobre elas na sua ausência. }\end{array}$ \\
\hline Memória Episódica & Recontando histórias (6) & $\begin{array}{l}\text { Ler uma história dividida em quatro trechos. Recontar a } \\
\text { história completa, incluindo o máximo de informações. }\end{array}$
\end{tabular}

Memorização de nomes (8) Memorizar nomes associados a faces e, em seguida, identificar os nomes correspondentes às faces.

Memorização de números (6) Memorizar e repetir uma sequência numérica.

Memória de Trabalho Dominó Fonológico (10) Ouvir uma palavra e gerar, na sequência, outra que inicie com a última sílaba da palavra ouvida.

Pa-pa-ra-pa-pá (9) Entoar um ritmo e, simultaneamente, contar mentalmente o número de estímulos-alvo em um conjunto de distratores.

Sequência de histórias (4) Ler trechos separados de uma história. Em seguida, reproduzir a história organizando os trechos na ordem correta.

Meses (12)

Ouvir uma sequência de meses e organizá-la em ordem cronológica.

Letras (15)

Contar mentalmente o número de letras de um conjunto de palavras.

Para submissão dos itens à avaliação dos especialistas, foi elaborado um formulário, enviado e respondido no formato on-line, contendo, para cada tarefa, as instruções, um exemplo de item e a informação sobre quais habilidades cognitivas pretendia-se alcançar com a tarefa. A escala de respostas foi composta por quatro possibilidades de avaliação: 1. A tarefa não representa adequadamente as habilidades pretendidas; 2 . A tarefa representa parcialmente as habilidades pretendidas; 3 . A tarefa representa adequadamente as habilidades pretendidas. Para as opções 1 e 2, solicitou-se que o respondente identificasse outras habilidades cognitivas tão ou mais demandadas pela tarefa.

\section{Resultados}

A opção 2 (A tarefa representa parcialmente as habilidades pretendidas) não foi escolhida em nenhum dos itens, portanto, considerou-se apenas as opções 1 e 3. A opção 1 (A tarefa não representa adequadamente as habilidades pretendidas) foi computada com escore 0 e a opção 3 (A tarefa representa adequadamente as habilidades pretendidas) foi computada com escore 1. A Tabela 2 apresenta os resultados da avaliação de cada tarefa por meio da porcentagem de concordância com adequação da tarefa à habilidade pretendida, assim como as sugestões de habilidades tão ou mais demandadas pela tarefa.
Das treze tarefas avaliadas, apenas onze alcançaram uma avaliação superior a 70\% (considerado adequado de acordo com Urbina, 2007) quanto à adequação na representação das habilidades pretendidas: Labirintos, Figuras, Estimulação Sonora, Recontando Histórias, Busca Visual, Visualizando Figuras, Dominó Fonológico, Sequência de Histórias, Pa-pa-ra-pa-pá e Letras. As tarefas com menor consenso na avaliação $(68,75 \%)$ quanto à adequação foram Memorização de Nomes e Memorização de Números, para as quais a maior parte dos juízes sugeriu como habilidade mais demandada a memória de curto prazo.

Finalmente, o cálculo do índice Kappa foi efetuado utilizando-se o software R (R Core Team, 2012) com o pacote Inter-rater reliability (Gamer, Lemon, Fellows, \& Singh, 2012) que, por meio da função intraclass correlation coeficient (ICC), fornece o índice Fleiss Kappa, adequado para mais de dois avaliadores. $\mathrm{O}$ resultado apontou um índice de concordância baixo $(-0,02<\mathrm{ICC}<0,12)$ com valor $p=0,18$

\section{Estudo de Validade Estrutural}

Para a verificação da replicabilidade do modelo teórico proposto para determinação das habilidades cognitivas treinadas na intervenção, efetuou-se uma análise fatorial aplicada às tarefas do treino. Esse estudo também buscou verificar modelos alternativos, de forma a comparar a viabilidade tanto do modelo teórico, quanto de modelos concorrentes. 
Tabela 2

Avaliação dos Juízes sobre Representação das Tarefas às Habilidades Pretendidas

\begin{tabular}{|c|c|c|c|}
\hline Tarefa & $\begin{array}{l}\text { Habilidade } \\
\text { Pretendida }\end{array}$ & $\begin{array}{l}\text { Concordaram } \\
\text { com adequação } \\
\text { da tarefa (\%) }\end{array}$ & $\begin{array}{l}\text { Outras habilidades } \\
\text { sugeridas }\end{array}$ \\
\hline Labirintos & $\begin{array}{l}\text { Atenção e } \\
\text { Velocidade de } \\
\text { Processamento }\end{array}$ & $75 \%(n=12)$ & $\begin{array}{l}\text { - Habilidade visuoespacial } \\
\text { - Planejamento } \\
\text { - Coordenação motora } \\
\text { - Flexibilidade cognitiva }\end{array}$ \\
\hline Figuras & $\begin{array}{l}\text { Habilidades } \\
\text { perceptomotoras }\end{array}$ & $75 \%(n=12)$ & $\begin{array}{l}\text { - Memória de curto prazo } \\
\text { - Habilidades mnemônicas } \\
\text { - Memória figurativa } \\
\text { - Memória visuoespacial }\end{array}$ \\
\hline Estimulação Sonora & $\begin{array}{l}\text { Atenção para } \\
\text { estímulos auditivos }\end{array}$ & $75 \%(n=12)$ & $\begin{array}{l}\text { - Habilidade de identificação lexical } \\
\text { - Vocabulário } \\
\text { - Memória semântica }\end{array}$ \\
\hline Recontando histórias & Memória episódica & $93,7 \%(n=15)$ & Não houve sugestão \\
\hline Busca Visual & $\begin{array}{l}\text { Atenção e velocidade } \\
\text { de processamento }\end{array}$ & $87,5 \%(n=14)$ & Rapidez cognitiva \\
\hline Visualizando Figuras & Memória visual & $93,7 \%(n=15)$ & Memória episódica \\
\hline Memorização de nomes & Memória episódica & $68,75 \%(n=11)$ & $\begin{array}{l}\text { - Memória de curto prazo } \\
\text { - Memória de trabalho } \\
\text { - Memória de faces }\end{array}$ \\
\hline Memorização de números & Memória episódica & $68,75 \%(n=11)$ & $\begin{array}{l}\text { - Memória de curto prazo } \\
\text { - Memória de trabalho }\end{array}$ \\
\hline Dominó Fonológico & Memória de trabalho & $75 \%(n=12)$ & $\begin{array}{l}\text { - Consciência fonológica } \\
\text { - Fluência verbal }\end{array}$ \\
\hline Pa-pa-ra-pa-pá & Memória de trabalho & $75 \%(n=12)$ & $\begin{array}{l}\text { - Atenção dividida } \\
\text { - Controle inibitório }\end{array}$ \\
\hline Sequência de histórias & Memória de trabalho & $87,5 \%(n=14)$ & Não houve sugestão \\
\hline Meses & Memória de trabalho & $93,7 \%(n=15)$ & Não houve sugestão \\
\hline Letras & Memória de trabalho & $75 \%(n=12)$ & Não houve sugestão \\
\hline
\end{tabular}

\section{Participantes}

A amostra foi composta por um grupo de 47 idosos que receberam o treino cognitivo (média de idade $=69,66$ anos, $\mathrm{DP}=7,51$; média de escolarização $=7,40$ anos, $\mathrm{DP}=4,86)$, tendo sido este o grupo experimental de um estudo prévio, realizado para verificar o impacto da intervenção no desempenho mental de idosos (Santos, 2015). Os idosos foram recrutados da comunidade e passaram por uma triagem, na qual alguns critérios de inclusão foram aplicados: (a) Ter mais de 60 anos; (b) Ausência de déficit sensorial ou motor grave; (c) Ausência de diagnóstico de quadro demencial; (d) Ausência de diagnóstico de transtorno psiquiátrico.

\section{Instrumentos}

\section{Treino Cognitivo}

A intervenção consistiu em 12 sessões individuais, com duração de cerca de 90 minutos cada e frequência semanal. Foram aplicadas 13 tarefas (ver Tabela 1), totalizando 115 itens conduzidos de acordo com diretrizes de intervenção. Tais itens foram precedidos, na mesma quantidade, de itens de teste, com mesma tarefa e conteúdo similar, consistindo em um pré-treino sem orientações de intervenção. A análise fatorial foi conduzida apenas com esse conjunto de itens de teste. $\mathrm{O}$ material do programa de treino consistiu em três cadernos, de uso individual: 1. Cadernos de Instrução (material elaborado para os aplicadores com instruções detalhadas sobre os procedimentos de condução de cada sessão); 2. Caderno de Estímulos (conjunto de estímulos apresentados aos participantes); 3. Protocolo de Registros, elaborado para registrar o desempenho do participante em todos os itens das tarefas do treino. Para informações mais detalhadas sobre o treino cognitivo, ver Santos (2015).

\section{Procedimentos}

Após a construção do treino cognitivo, um estudo experimental inicial foi conduzido para verificação do impacto da intervenção sobre o desempenho cognitivo da amostra. Participaram dessa etapa um grupo de 80 idosos, dividido em grupo experimental $(n=47)$, que recebeu o treino, e grupo controle $(n=33)$. Foram utilizadas, como medidas cognitivas pré e pós-intervenção, em ambos os grupos: Teste de Memória Episódica (Yassuda et al., 2006) e seis subtestes da Escala Wechsler de Inteligência para Adultos WAIS-III (Nascimento, 
2004): Completar Figuras, Códigos, Aritmética, Raciocínio Matricial, Dígitos e Procurar Símbolos. Os resultados do teste Rank transformation ANOVA indicaram que houve efeito significativo de treino, por meio da interação entre os fatores Tempo versus Grupo, para as medidas: Completar Figuras $(\mathrm{F}(74)=14,88, p=0,0002$, $d=0,90$, cles $=73,69 \%)$, Códigos $(\mathrm{F}(74)=5,66, p=0,019$, $d=0,55$, cles $=65,21 \%)$ e Dígitos $(\mathrm{F}(74)=5,38, p=0,02$, $d=0,54$, cles $=64,85 \%$ ).

Para a condução do presente estudo, efetuou-se a análise dos protocolos de registros individuais do grupo experimental. Após digitação dos dados, alguns procedimentos para organização dos dados foram adotados: 1. Para os itens não realizados em virtude da aplicação dos critérios de interrupção, atribuiu-se pontuação de erro; 2. Exclusão das tarefas Estimulação Sonora e Dominó Fonológico, devido à baixa discriminação dos itens (quase todos os participantes alcançaram pontuação máxima nas tarefas). Após tal exclusão, foram analisadas 12 tarefas, totalizando 95 itens; 3 . Em virtude do número proporcionalmente grande de variáveis $(n=95)$ para o número de observações $(n=47)$ para fins de se efetuar a análise fatorial, foram computados os escores brutos de cada tarefa, totalizando, assim, 12 itens (ao invés de 95). Ressalta-se que a tarefa Cópia foi transformada em dois escores, cada um com o somatório de três itens específicos dessa tarefa.

\section{Análise de Dados}

Considerando o tamanho da amostra do estudo $(n=47)$, efetuou-se uma análise fatorial bayesiana. As estatísticas bayesianas permitem incorporar um background anterior de conhecimentos nas análises (ao invés de testar a mesma hipótese nula várias vezes) e não confia na teoria das grandes amostras. Assim, atua melhor em amostras pequenas $(N<200)$, sendo tipicamente aplicada em estudos clínicos (Lee \& Shi, 2000; Lee \& Song, 2004). Ao invés da utilização do método maximum likelihood (recomendado para amostras com
N>200), utilizou-se um conjunto de métodos denominado Markov Chains Monte Carlo Methods (MCMC), que torna possível amostrar densidades complexas e de alta dimensão. Tais métodos envolvem a simulação de uma cadeia de Markov construída que converge para a densidade de interesse (normalmente, a densidade posterior) (Press, 2009). Todas as análises foram efetuadas utilizando-se o software MPLUS Versão 7 (Muthén \& Muthén, 2012).

Para a análise da viabilidade do modelo testado, foi utilizado o Posterior Predictive Checking (PPC; Gelman \& Hill, 2006). O que o PPC faz é basicamente simular dados replicados a partir de um determinado modelo, e comparar esses dados simulados com os dados observados (Gelman \& Hill, 2006), por meio da distribuição de um índice estatístico $f$, que é baseado em um teste de qui-quadrado da razão da verossimilhança (Muthén \& Asparouhov, 2012). O que se busca é verificar as discrepâncias sistemáticas entre os dados simulados e os observados, a partir de um determinado modelo. De forma a se verificar o ajuste do modelo aos dados, utiliza-se o Posterior Predictive P-value (PPP), dentro da abordagem do PPC. Valores muito baixos de PPP indicam que o modelo não se ajusta aos dados (Muthén \& Asparouhov, 2012). Um modelo apresenta um ajuste considerado excelente quando o valor do PPP for próximo ou igual a 0,5 , e quando o valor zero da diferença do índice estatístico $f$ nos dados observados e simulados incide no meio da distribuição do intervalo de confiança da distribuição do índice $f$ (Muthén \& Asparouhov, 2012). O modelo teórico com quatro fatores (1. Atenção e Velocidade de Processamento; 2. Memória Visual; 3. Memória Episódica; 4. Memória de Trabalho) foi comparado com outros doze modelos.

\section{Resultados}

A Tabela 3 apresenta a estrutura do modelo teórico e de outros doze modelos testados, seguidos do índice de ajuste.

Tabela 3

Estrutura e Ajuste dos 13 Modelos Testados

\begin{tabular}{lll}
\hline \multicolumn{1}{c}{ Modelo } & \multicolumn{1}{c}{ Variáveis Latentes } & \multicolumn{1}{c}{ Variáveis Observadas } \\
\hline Modelo Unidimensional & Capacidade Geral & Lab, BV, PA, HI, Me, Le, Cop1, Cop2, VF, RC, MN, Mnum; 0,000 \\
\hline Modelo com & Atenção/Velocidade e Memória & Cop1, Cop2, VF, RC, MN, Mnum; \\
Dois Fatores & Percepção e Memória Episódica/Visual & Cop1, Cop2, VF, RC, MN, Mnum; \\
\hline Modelo com & Atenção/Velocidade e Memória & Lab, BV, PA, HI, Me, Le; \\
Três Fatores & Capacidade Perceptual & Cop1, Cop2; \\
& Memória Episódica/Visual & VF, RC, MN, Mnum; \\
\hline Modelo com & Atenção/Velocidade & Lab, BV; \\
Quatro Fatores & Capacidade Perceptual & Cop1, Cop2; \\
& Memória Episódica/Visual & VF, RC, MN, Mnum; \\
& Memória de Trabalho & PA, HI, Me, Le; \\
\hline
\end{tabular}


Tabela 3 (continuação)

Estrutura e Ajuste dos 13 Modelos Testados

\begin{tabular}{|c|c|c|c|}
\hline Modelo & Variáveis Latentes & Variáveis Observadas & PPP \\
\hline \multirow{4}{*}{$\begin{array}{l}\text { Modelo com Quatro } \\
\text { Fatores (Teórico) }\end{array}$} & Atenção/Velocidade & Lab, BV; & 0,020 \\
\hline & Memória Visual & Cop1, Cop2, VF; & \\
\hline & Memória Episódica & RC, MN, Mnum; & \\
\hline & Memória de Trabalho & PA, HI, Me, Le; & \\
\hline \multirow{5}{*}{$\begin{array}{l}\text { Modelo com } \\
\text { Cinco Fatores }\end{array}$} & Atenção/Velocidade & Lab, BV; & 0,017 \\
\hline & Memória Visual & Cop1, Cop2, VF; & \\
\hline & Memória Episódica & MN, Mnum; & \\
\hline & Memória de Trabalho & PA, Me, Le; & \\
\hline & Linguagem & RC, HI; & \\
\hline \multirow{2}{*}{$\begin{array}{l}\text { Modelo Bifatorial com } \\
\text { Dois Fatores Específicos }\end{array}$} & Fator Geral & Lab, BV, PA, HI, Me, Le, Cop1, Cop2, VF, RC, MN, Mnum; & 0,032 \\
\hline & Atenção/Velocidade e Memória & Lab, BV, PA, HI, Me, Le; & \\
\hline \multirow{3}{*}{$\begin{array}{l}\text { Modelo Bifatorial com } \\
\text { Três Fatores Específicos }\end{array}$} & Fator Geral & Lab, BV, PA, HI, Me, Le, Cop1, Cop2, VF, RC, MN, Mnum; & 0,25 \\
\hline & Atenção/Velocidade e Memória & Lab, BV, PA, HI, Me, Le; & \\
\hline & Capacidade Perceptual & Cop1, Cop2; & \\
\hline \multirow{5}{*}{$\begin{array}{l}\text { Modelo Bifatorial com } \\
\text { Quatro Fatores Específicos }\end{array}$} & Fator Geral & Lab, BV, PA, HI, Me, Le, Cop1, Cop2, VF, RC, MN, Mnum; & 0,21 \\
\hline & Atenção/Velocidade & Lab, BV; & \\
\hline & Capacidade Perceptual & Cop1, Cop2; & \\
\hline & Memória Episódica/Visual & VF, RC, MN, Mnum; & \\
\hline & Memória de Trabalho & PA, HI, Me, Le; & \\
\hline \multirow{5}{*}{$\begin{array}{l}\text { Modelo Bifatorial com } \\
\text { Quatro Fatores Específicos } \\
\text { (Teórico) }\end{array}$} & Fator Geral & Lab, BV, PA, HI, Me, Le, Cop1, Cop2, VF, RC, MN, Mnum; & 0,162 \\
\hline & Atenção/Velocidade & Lab, BV; & \\
\hline & Memória Visual & Cop1, Cop2, VF; & \\
\hline & Memória Episódica & RC, MN, Mnum; & \\
\hline & Memória de Trabalho & PA, HI, Me, Le; & \\
\hline \multirow{6}{*}{$\begin{array}{l}\text { Modelo Bifatorial com } \\
\text { Cinco Fatores Específicos }\end{array}$} & Fator Geral & Lab, BV, PA, HI, Me, Le, Cop1, Cop2, VF, RC, MN, Mnum; & 0,12 \\
\hline & Atenção/Velocidade & $\mathrm{Lab}, \mathrm{BV}$ & \\
\hline & Memória Visual & Cop1, Cop2, VF; & \\
\hline & Memória Episódica & MN, Mnum; & \\
\hline & Memória de Trabalho & PA, Me, Le; & \\
\hline & Linguagem & RC, HI; & \\
\hline \multirow{5}{*}{$\begin{array}{l}\text { Modelo Bifatorial } \\
\text { com Quatro } \\
\text { Fatores - Modificado } 1\end{array}$} & Fator Geral & Lab, BV, PA, HI, Me, Le, Cop1, Cop2, VF, RC, MN, Mnum; & 0,305 \\
\hline & f1 & RC, VF, BV, Lab; & \\
\hline & f2 & Cop1, Cop2; & \\
\hline & f3 & Mnum, MN, PA; & \\
\hline & f4 & Le, Me; & \\
\hline \multirow{6}{*}{$\begin{array}{l}\text { Modelo Bifatorial } \\
\text { com Cinco } \\
\text { Fatores - Modificado } 1\end{array}$} & Fator Geral & Lab, BV, PA, HI, Me, Le, Cop1, Cop2, VF, RC, MN, Mnum; & 0,330 \\
\hline & f1 & $\mathrm{RC}, \mathrm{VF}$ & \\
\hline & f2 & Cop1, Cop2; & \\
\hline & f3 & Mnum, MN, PA; & \\
\hline & f4 & Le, Me; & \\
\hline & f5 & BV, Lab, HI; & \\
\hline \multirow{8}{*}{$\begin{array}{l}\text { Modelo Bifatorial com } \\
\text { Cinco Fatores Específicos } \\
\text { e Dois Fatores de Segunda } \\
\text { Ordem }\end{array}$} & Fator Geral & Lab, BV, PA, HI, Me, Le, Cop1, Cop2, VF, RC, MN, Mnum; & 0,389 \\
\hline & f1 & $\mathrm{RC}, \mathrm{VF}$ & \\
\hline & f2 & Cop1, Cop2; & \\
\hline & f3 & Mnum, MN, PA; & \\
\hline & $\mathrm{f} 4$ & Le, Me; & \\
\hline & f5 & BV, Lab, HI; & \\
\hline & Memória Episódica & $\mathrm{f} 1, \mathrm{f} 2$ & \\
\hline & Memória de Trabalho & $\mathrm{f} 3, \mathrm{f} 5$ & \\
\hline
\end{tabular}

Nota. Lab=Labirintos; BV=Busca Visual; PA=Pa-pa-ra-pa-pá; HI=História; Me=Meses; Le=Letras; Cop1=Cópias (itens 1-3); Cop2= Cópias (itens 4-6); VF=Visualizando Figuras; RC=Recontando Histórias; MN=Memorização de Nomes; MNum=Memorização de Números 
É possível observar que, dos treze modelos testados, apenas cinco apresentaram PPP igual ou superior a 0,20 : modelo bifatorial com três fatores específicos $(\mathrm{PPP}=0,25)$, modelo bifatorial com quatro fatores específicos $(\mathrm{PPP}=0,21)$, modelo bifatorial com quatro fatores específicos (modificado 1) $(\mathrm{PPP}=0,305)$, modelo bifatorial com cinco fatores (modificado 1$)(\mathrm{PPP}=0,33)$ e o modelo bifatorial com cinco fatores específicos e dois fatores de segunda ordem ( $\mathrm{PPP}=0,389)$. Este último modelo apresenta cinco fatores específicos que correspondem às variáveis latentes: Memória Visual (fator 1), Habilidades Perceptomotoras (fator 2), Memória Episódica (fator 3), Memória de Trabalho (fator 4), Atenção/Velocidade (fator 5), além do fator geral que explica diretamente os escores somados nas tarefas, e dois fatores de segunda ordem, sendo um genericamente denominado de Memória de Trabalho e o outro, de Memória Episódica.

A Figura 1 apresenta a distribuição da diferença da estatística $f$ entre os dados observados e os simulados.

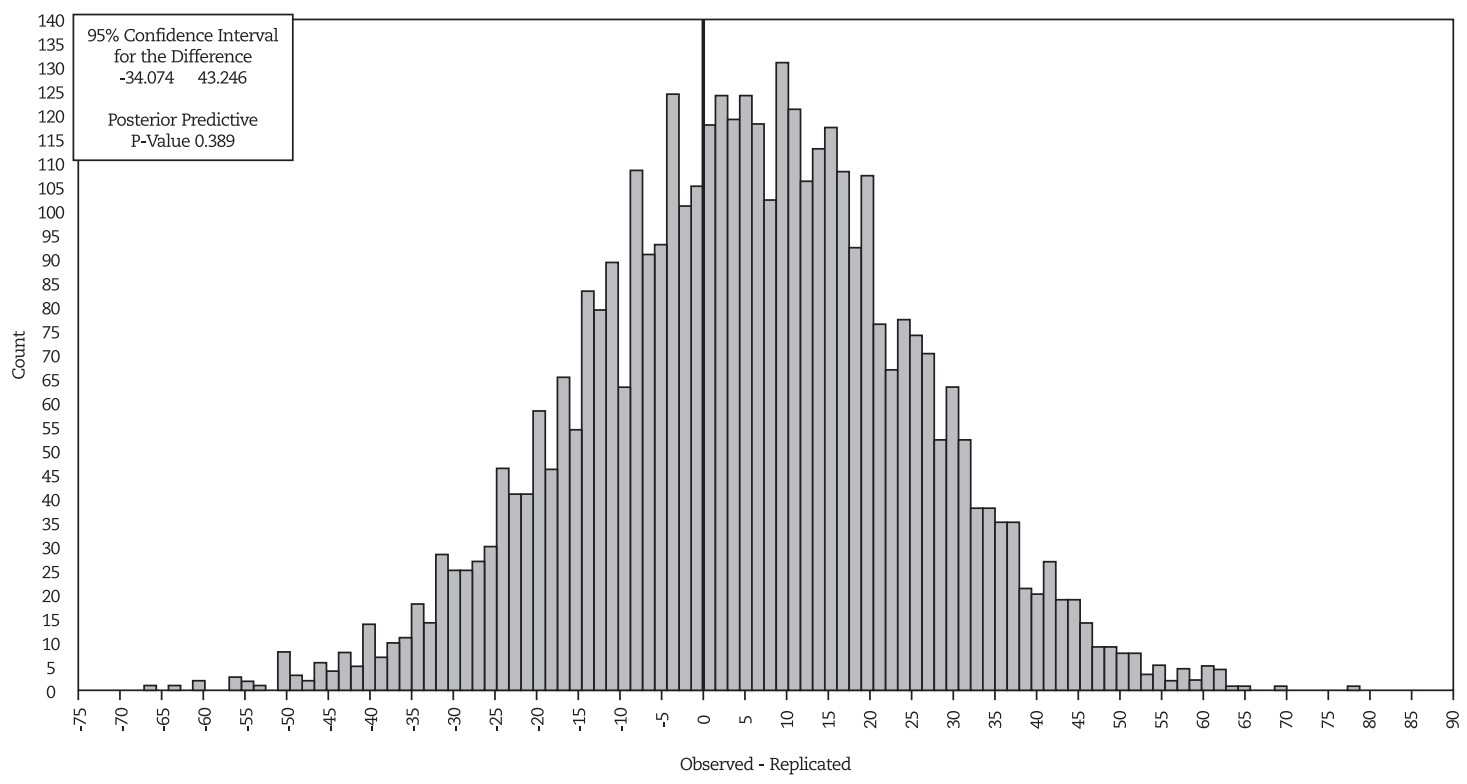

Figura 1. Distribuição de $f$ entre dados observados e simulados

É possível verificar que o valor de zero da diferença incide quase no meio da distribuição. Aliado ao valor relativamente alto do PPP $(0,389)$, a distribuição da diferença do índice estatístico $f$ permite apontar que o modelo bifatorial com cinco fatores específicos e dois fatores de segunda ordem apresenta um ajuste adequado aos dados. A Figura 2 mostra esse modelo e as cargas padronizadas.

Já a Tabela 4 mostra os valores das cargas padronizadas do modelo final, assim como o desvio padrão e o intervalo de confiança de $95 \%$ das cargas.

\section{Discussão}

Como melhorar a cognição na velhice é uma questão que tem sido ativamente considerada na sociedade. Constantemente depara-se com conselhos de nutrição e prática de saúde para manter o cérebro e a mente saudáveis, ou com uma vasta literatura sobre como manter a memória na velhice, uma das habilidades mais vulneráveis da cognição (ex.: Alvarez, 2005; Einstein \&
McDaniel, 2004). A despeito das inúmeras iniciativas que prometem oferecer um aumento no desempenho mental, há uma tendência entre os pesquisadores da área de tecer avaliações mais críticas e racionais sobre as possibilidades e limitações das intervenções, impondo especificações metodológicas cada vez mais rigorosas para investigação da eficácia dos treinos. Um exemplo dessa iniciativa é o Consort - Consolidated Standards of Reporting Trials (http://www.consort-statement.org), possibilitando a condução de estudos robustos de meta-análise e o alcance de inferências sobre a questão fundamental que permanece dividindo a área: é possível alcançar mudanças estruturais e permanentes na cognição por meio dos treinos cognitivos? A investigação dessa questão é feita, predominantemente, por meio da verificação do impacto que as intervenções geram no desempenho cognitivo do indivíduo. Observa-se, contudo, uma lacuna nas literaturas internacional e nacional no que diz respeito à investigação da validade das intervenções para assegurar que as habilidades cognitivas pretendidas estão, de fato, sendo estimuladas. 


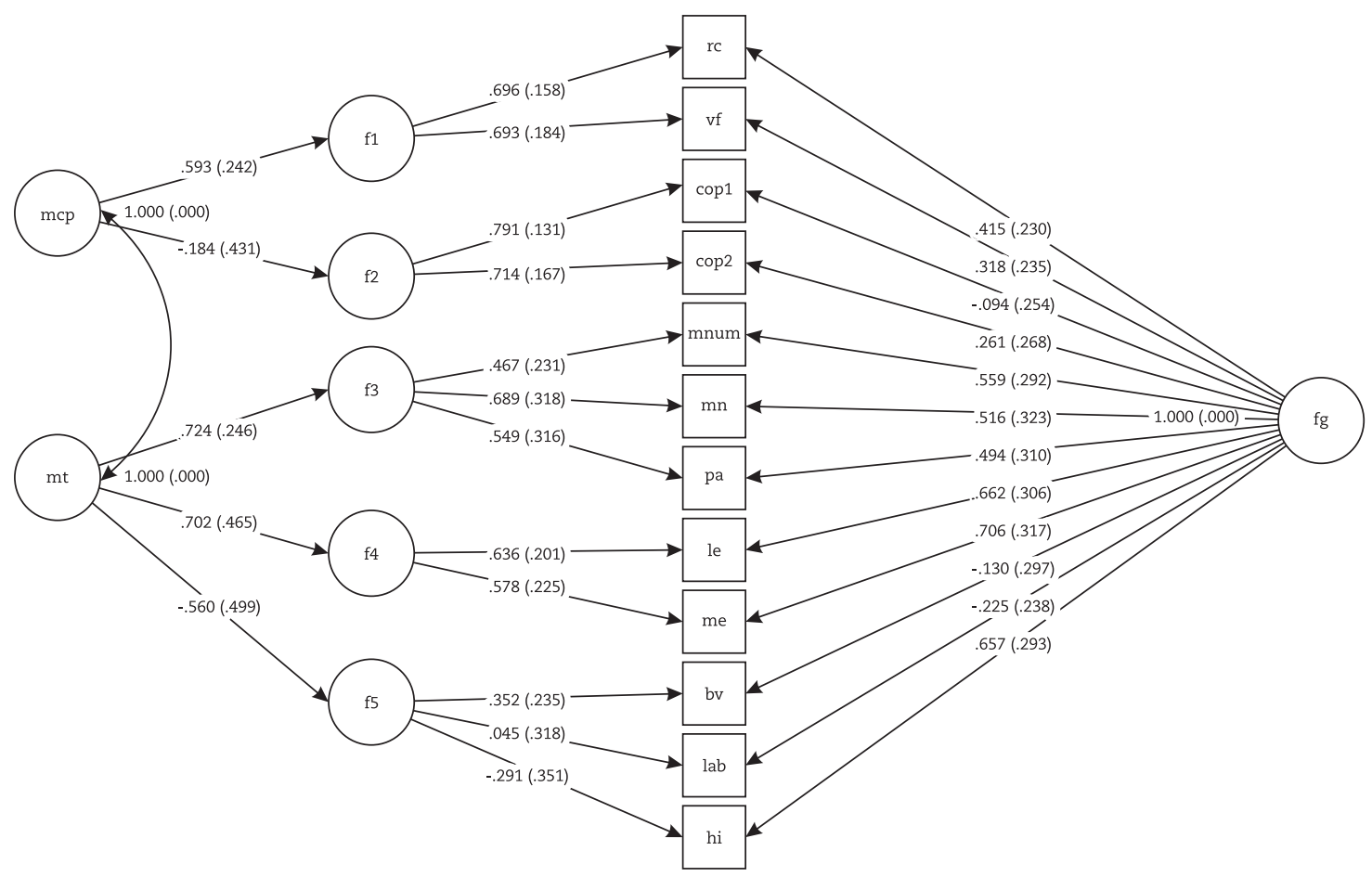

Figura 2. Modelo final com melhor ajuste

Tabela 4

Cargas Padronizadas do Modelo Final

\begin{tabular}{|c|c|c|c|c|c|c|}
\hline \multirow{2}{*}{ Variável Latente } & \multirow{2}{*}{ Variável Observada } & \multirow{2}{*}{$\begin{array}{c}\text { Carga } \\
\text { Padronizada }\end{array}$} & \multirow{2}{*}{ DP } & \multirow{2}{*}{$\mathrm{P}$-value } & \multicolumn{2}{|c|}{$\begin{array}{c}\text { Intervalo de } \\
\text { confiança (95\%) }\end{array}$} \\
\hline & & & & & $\begin{array}{c}\text { Lower } \\
2,5 \% \\
\end{array}$ & $\begin{array}{c}\text { Upper } \\
2,5 \% \\
\end{array}$ \\
\hline \multirow[t]{12}{*}{ Fator Geral } & Lab & $-0,23$ & 0,24 & 0,18 & $-0,61$ & 0,33 \\
\hline & Cop1 & $-0,09$ & 0,25 & 0,36 & $-0,52$ & 0,47 \\
\hline & Cop2 & 0,26 & 0,27 & 0,16 & $-0,27$ & 0,78 \\
\hline & $\mathrm{RC}$ & 0,42 & 0,23 & 0,06 & $-0,15$ & 0,77 \\
\hline & BV & $-0,13$ & 0,30 & 0,36 & $-0,56$ & 0,55 \\
\hline & VF & 0,32 & 0,24 & 0,11 & $-0,23$ & 0,70 \\
\hline & MN & 0,52 & 0,32 & 0,10 & $-0,35$ & 0,86 \\
\hline & MNum & 0,56 & 0,29 & 0,06 & $-0,33$ & 0,85 \\
\hline & $\mathrm{PA}$ & 0,49 & 0,31 & 0,09 & $-0,35$ & 0,84 \\
\hline & $\mathrm{Me}$ & 0,71 & 0,32 & 0,05 & $-0,38$ & 0,95 \\
\hline & HI & 0,66 & 0,29 & 0,05 & $-0,41$ & 0,90 \\
\hline & Le & 0,66 & 0,31 & 0,05 & $-0,35$ & 0,92 \\
\hline \multirow{2}{*}{$\begin{array}{l}\text { Fator } 1 \\
\text { (Memória Visual) }\end{array}$} & $\mathrm{RC}$ & 0,70 & 0,16 & 0,00 & 0,36 & $0,95^{*}$ \\
\hline & VF & 0,69 & 0,18 & 0,00 & 0,28 & $0,96^{*}$ \\
\hline \multirow{2}{*}{$\begin{array}{l}\text { Fator } 2 \\
\text { (Habilidades perceptomotoras) }\end{array}$} & COP1 & 0,79 & 0,13 & 0,00 & 0,49 & $0,98^{*}$ \\
\hline & COP2 & 0,71 & 0,17 & 0,00 & 0,33 & $0,96^{*}$ \\
\hline \multirow{3}{*}{$\begin{array}{l}\text { Fator } 3 \\
\text { (Memória Episódica) }\end{array}$} & MNum & 0,47 & 0,21 & 0,00 & 0,04 & $0,82^{*}$ \\
\hline & $\mathrm{MN}$ & 0,69 & 0,32 & 0,06 & $-0,34$ & 0,96 \\
\hline & PA & 0,55 & 0,32 & 0,09 & $-0,43$ & 0,86 \\
\hline \multirow{2}{*}{$\begin{array}{l}\text { Fator } 4 \\
\text { (Memória de Trabalho) }\end{array}$} & LE & 0,64 & 0,20 & 0,00 & 0,20 & $0,94^{*}$ \\
\hline & $\mathrm{ME}$ & 0,58 & 0,23 & 0,01 & 0,08 & $0,93^{*}$ \\
\hline
\end{tabular}


Tabela 4 (continuação) Cargas Padronizadas do Modelo Final

\begin{tabular}{|c|c|c|c|c|c|c|}
\hline \multirow{2}{*}{ Variável Latente } & \multirow{2}{*}{ Variável Observada } & \multirow{2}{*}{$\begin{array}{c}\text { Carga } \\
\text { Padronizada }\end{array}$} & \multirow{2}{*}{$\mathrm{DP}$} & \multirow{2}{*}{ P-value } & \multicolumn{2}{|c|}{$\begin{array}{c}\text { Intervalo de } \\
\text { confiança (95\%) }\end{array}$} \\
\hline & & & & & $\begin{array}{c}\text { Lower } \\
2,5 \%\end{array}$ & $\begin{array}{l}\text { Upper } \\
2,5 \%\end{array}$ \\
\hline \multirow{3}{*}{$\begin{array}{l}\text { Fator } 5 \\
\text { (Atenção/Velocidade) }\end{array}$} & BV & 0,35 & 0,24 & 0,00 & 0,03 & $0,86^{*}$ \\
\hline & LAB & 0,05 & 0,32 & 0,44 & $-0,66$ & 0,62 \\
\hline & $\mathrm{HI}$ & $-0,29$ & 0,35 & 0,23 & $-0,84$ & 0,49 \\
\hline \multirow[t]{2}{*}{ Memória Episódica } & Fator 1 & 0,60 & 0,24 & 0,00 & 0,12 & $0,98^{*}$ \\
\hline & Fator 2 & $-0,18$ & 0,43 & 0,35 & $-0,89$ & 0,79 \\
\hline \multirow[t]{3}{*}{ Memória Trabalho } & Fator 3 & 0,72 & 0,25 & 0,00 & 0,10 & $0,98^{*}$ \\
\hline & Fator 4 & 0,70 & 0,47 & 0,14 & $-0,75$ & 0,98 \\
\hline & Fator 5 & $-0,56$ & 0,50 & 0,21 & $-0,97$ & 0,83 \\
\hline \multicolumn{7}{|c|}{ Correlação } \\
\hline \multirow[t]{7}{*}{ Fator Geral } & F1 & 0,00 & 0,00 & 1,00 & 0,00 & 0,00 \\
\hline & $\mathrm{F} 2$ & 0,00 & 0,00 & 1,00 & 0,00 & 0,00 \\
\hline & F3 & 0,00 & 0,00 & 1,00 & 0,00 & 0,00 \\
\hline & $\mathrm{F} 4$ & 0,00 & 0,00 & 1,00 & 0,00 & 0,00 \\
\hline & F5 & 0,00 & 0,00 & 1,00 & 0,00 & 0,00 \\
\hline & Memória Episódica & 0,00 & 0,00 & 1,00 & 0,00 & 0,00 \\
\hline & Memória de Trabalho & 0,00 & 0,00 & 1,00 & 0,00 & 0,00 \\
\hline Memória de Trabalho & Memória Episódica & 0,13 & 0,47 & 0,40 & $-0,87$ & 0,90 \\
\hline
\end{tabular}

A presente pesquisa buscou inserir o seguinte questionamento: considerando que os autores das intervenções, em geral, baseiam-se nos seus pressupostos teóricos e na revisão da literatura para formulação das tarefas, como assegurar que a intervenção está, de fato, alcançando as habilidades reportadas? Como alternativa para a investigação dessa questão, a presente pesquisa propôs a condução de dois estudos de validade, tipicamente realizados no campo da mensuração, mas escassamente incorporados para investigação de programas de intervenção: um estudo de validade de conteúdo e um estudo de validade estrutural. Dessa forma, argumenta-se ser possível investigar a adequação das tarefas do treino às habilidades-alvo pretendidas e confirmar, assim, o modelo teórico subjacente à intervenção.

O estudo de validade de conteúdo apresentou resultado baixo quanto à concordância dos especialistas (índice Fleiss Kappa $=0,11 ;-0,02<$ icc $<0,12, p=0,18$ ). Contudo, analisando as concordâncias individuais de cada tarefa, observa-se, de acordo com Urbina (2007): 1. uma alta concordância $(>80 \%)$ sobre a adequação dos itens do treino para cinco das treze tarefas: Recontando Histórias (93,7\%), Busca Visual (87,5\%), Visualizando Figuras (93,7\%), Sequência de Histórias $(87,5 \%)$ e Meses $(93,7 \%)$; 2 . uma concordância moderada $(=75 \%)$ para cinco tarefas: Labirintos, Figuras, Estimulação Sonora, Dominó Fonológico, Pa-pa-ra-papá e Letras; e 3. um baixo consenso (<70\%) na avaliação das tarefas Memorização de Nomes e Memorização de Números, para as quais predominaram as sugestões de que a tarefa demanda memória de curto prazo (sugestão predominante) e memória de trabalho.

Foi possível observar, analisando-se as sugestões dos juízes, a presença de certa divergência no uso de termos para definir uma mesma habilidade cognitiva (Ex.: velocidade de processamento versus rapidez cognitiva; identificação lexical versus vocabulário), a indicação de habilidades cognitivas tipicamente associadas a outras fases do desenvolvimento (Ex.: consciência fonológica, predominantemente demandada na aprendizagem de leitura e escrita) e para as habilidades mnemônicas, observou-se a identificação de domínios associados ao critério temporal (memória de curto prazo) em detrimento do critério conteúdo (memória episódica).

O campo de investigação da cognição/inteligência é marcado pela coexistência de modelos teóricos diversos que culminam, naturalmente, em uma diversidade taxonômica que dificulta a condução de estudos de validade de conteúdo. Uma alternativa para amenizar esse impacto é especificar, no questionário do avaliador, a perspectiva teórica adotada pelo autor no entendimento de uma dada habilidade, explicitando sua definição. Outra possibilidade é reunir um grupo de especialistas de uma mesma área (tais como: Avaliação Psicológica, Psicologia do Desenvolvimento Humano, Psicologia Cognitiva, Neurociências), ao invés de formar um grupo proveniente de áreas afins para assegurar uma maior uniformidade no uso dos termos. A primeira estratégia foi adotada nesta pesquisa, porém, o grupo de juízes participantes do estudo caracterizou-se por uma maior 
heterogeneidade de perspectivas teóricas, o que pode ter contribuído para a baixa concordância encontrada em algumas tarefas. Finalmente, é importante destacar que a baixa concordância geral (índice Fleiss-Kappa) sugere como hipóteses a ausência de evidências que sustentem a adequação do conteúdo das tarefas às habilidades cognitivas pretendidas, podendo ser necessária uma extensa revisão delas para aperfeiçoamento do programa. O resultado do índice, sensível ao tamanho amostra (Gamer et al., 2012), também pode sugerir a necessidade de ampliação da amostra de juízes.

O segundo estudo pretendeu verificar a replicabilidade do modelo teórico adotado para construção das tarefas do treino. Foram analisados os protocolos de registro individuais de um grupo de idosos submetido à intervenção $(n=47)$. O registro do desempenho dos participantes foi rigorosamente efetuado durante a intervenção, item por item, o que possibilitou a análise deles. Efetuou-se uma análise fatorial bayesiana, que revelou a estrutura fatorial subjacente às tarefas do programa. Após comparação entre treze modelos fatoriais, incluindo o modelo teórico, observou-se que o modelo bifatorial com cinco fatores específicos e dois fatores de segunda ordem $(\mathrm{PPP}=0,389)$ apresentou o melhor ajuste aos dados, superando o modelo teórico $(\mathrm{PPP}=0,162)$.

No modelo final (melhor ajuste), os autores concluíram que o domínio cognitivo definido como Fator 1 aproximou-se do domínio teórico Memória Visual, com a presença de uma tarefa planejada para estimular tal habilidade (Visualizando Figuras) e de uma segunda tarefa que pretendia, no modelo teórico, estimular a memória episódica (Recontando Histórias), mas que apresenta componentes de visualização mental. O Fator 2 , por sua vez, agrupou duas tarefas que pertenciam ao domínio Memória Visual no modelo teórico (Cópia 1 e Cópia 2), mas devido à natureza das tarefas foi denominado de Habilidades Perceptomotoras no modelo final. O Fator 3 agrupou duas das três tarefas elaboradas para estimular a Memória Episódica (Memorização de Nomes e Memorização de Números) e uma terceira tarefa elaborada para estimular memória de Trabalho. Considerando a predominância das tarefas de Memória Episódica nesse fator, ele foi assim denominado. Para o fator 4, agruparam-se duas das quatro tarefas elaboradas para estimular a Memória de Trabalho (Meses e Letras), sendo assim denominado tal fator. Finalmente, o fator cinco agrupou as duas tarefas elaboradas para estimular Atenção/Velocidade, e mais uma terceira tarefa planejada para estimular a Memória de Trabalho (Sequência de Histórias).

Identificados os fatores, é importante observar como as tarefas com alocação discordante entre os modelos teórico e empírico foram avaliadas pelos juízes quanto à concordância da sua adequação às habilidades pretendidas.

Tabela 5

Comparação entre os Resultados dos Estudos 1 e 2

\begin{tabular}{|c|c|c|c|c|}
\hline Tarefa & $\begin{array}{l}\text { Modelo } \\
\text { Teórico }\end{array}$ & $\begin{array}{l}\text { Modelo Final } \\
\text { (melhor ajuste) }\end{array}$ & $\begin{array}{l}\text { Coincidência } \\
\text { entre modelo } \\
\text { teórico e } \\
\text { empírico } \\
\end{array}$ & $\begin{array}{c}\text { \% de juízes que } \\
\text { concordaram } \\
\text { com a adequação } \\
\text { da tarefa }\end{array}$ \\
\hline Labirintos & $\begin{array}{c}\text { Atenção concentrada } \\
\text { e velocidade de } \\
\text { processamento }\end{array}$ & $\begin{array}{l}\text { Atenção concentrada } \\
\text { e velocidade de } \\
\text { processamento }\end{array}$ & SIM & $75 \%$ \\
\hline Recontando Histórias & Memória episódica & Memória visual & NÃO & $93,7 \%$ \\
\hline Busca Visual & $\begin{array}{l}\text { Atenção concentrada } \\
\text { e velocidade de } \\
\text { processamento }\end{array}$ & $\begin{array}{c}\text { Atenção concentrada } \\
\text { e velocidade de } \\
\text { processamento } \\
\end{array}$ & SIM & $85,7 \%$ \\
\hline Visualizando Figuras & Memória visual & Memória visual & SIM & $93,7 \%$ \\
\hline Pa-pa-ra-pa-pá & Memória de trabalho & Memória episódica & $\mathrm{NÃO}$ & $75 \%$ \\
\hline Meses & Memória de trabalho & Memória de trabalho & SIM & $93,7 \%$ \\
\hline Letras & Memória de trabalho & Memória de trabalho & SIM & $75 \%$ \\
\hline Cópias & Memória visual & $\begin{array}{c}\text { Habilidades } \\
\text { perceptomotoras }\end{array}$ & NÃO & $75 \%$ \\
\hline Memorização de Nomes & Memória episódica & Memória episódica & SIM & $68,75 \%$ \\
\hline Memorização de Números & Memória episódica & Memória episódica & SIM & $68,75 \%$ \\
\hline Sequência de Histórias & Memória de trabalho & Atenção/Velocidade & NÃO & $87,5 \%$ \\
\hline
\end{tabular}

Observa-se que, das onze tarefas analisadas, sete $(63,6 \%)$ apresentaram coincidência entre o modelo teórico e fatorial. Deste grupo, cinco foram avaliadas com mais de $70 \%$ de concordância pelos juízes e duas com $68,7 \%$ de concordância. Já para as quatro tarefas que não apresentaram coincidência entre o modelo teórico e 
o empírico, todas foram avaliadas com mais de $70 \%$ de concordância entre os juízes. A partir dessa análise qualitativa, pode-se concluir que não houve um padrão de convergência entre os resultados dos dois estudos.

Em suma, conclui-se que, de acordo com o modelo fatorial revelado, há evidências suportando que o treino cognitivo proposto alcançou estimulação predominante para as habilidades pretendidas pelo modelo teórico, porém, com uma concordância parcial das tarefas que compuseram cada domínio. Houve, também, a necessidade de inclusão, no modelo final, de um quinto fator
(Habilidades Perceptomotoras) como habilidade estimulada pela intervenção. É importante destacar, como limitação desta pesquisa, que não foi possível trabalhar com os dados diretamente dos itens devido à proporção de variáveis por observações. Para futuras pesquisas, sugere-se a ampliação do grupo de juízes do estudo de validade de conteúdo. Destaca-se a importância e urgência de um avanço metodológico da área durante a construção e testagem dos programas de intervenção, de modo a fornecer evidências robustas de validade das tarefas elaboradas.

\section{Referências}

Auffray, C., Juhel, J. (2001). General and differential effects of a multimodal cognitive training program for the elderly, Annee Psychologique, 101(1), 65-89. Recuperado de https://www.researchgate.net/publication/286396574_General_and_differential_effects_of_a_ multimodal_cognitive_training_program_for_the_eldenly

Alvarez, A (2005). Deu branco: Um guia para desenvolver o potencial da sua memória. Ed Record, São Paulo, S.P

Andrich, D. (2004). Controversy and the Rasch model: A characteristic of incompatible paradigms? Med Care, 42, I7-I16. doi: 10.1097/01. mlr.0000103528.48582.7c

Aramaki, F. O., \& Yassuda, M. S. (2011) Cognitive Training based on metamemory and mental images: follow-up evaluation and booster training effects. Dementia and Neuropsychology, 5(1), 48-53. Recuperado de http://www.demneuropsy.com.br/detalhe_artigo.asp?id=262

Bahar-Fuchs, A; Clare, L; \& Woods, B (2013). Cognitive training and cognitive rehabilitation for mild to moderate Alzheimer's disease and vascular dementia. The Cochrane Library, vol 6. doi: 10.1002/14651858.CD003260.pub2

Ball, K., Berch, D. B., Helmers, K. F., Jobe, J. B., Leveck, M. D., Marsiske, M., Morris, J. N.,Rebok, G. W., Smith, D. M., Teennstedt, S. L., Unverzagt, F. W., \& Willis, S.L., 2002. Effects of cognitive training interventions with older adults: a randomized controlled trial.JAMA, 288, 2271-2281. Recuperado de http://www.ncbi.nlm.nih.gov/pmc/articles/PMC2916176/pdf/nihms24855.pdf

Belleville, S., Gilbert, B., Fontaine, F., Gagnon, L., Ménard, É., \& Gauthier, S. (2006). Improvement of episodic memory in persons with mild cognitive impairment and healthy older adults: Evidence from a cognitive intervention program. Dementia and geriatric cognitive disorders, 22(5-6), 486-499.

Bergman-Nutley, S., So“derqvist, S., Bryde, S., Thorell, L. B., Humphreys, K., \& Klingberg, T. (2011). Gains in fluid intelligence after training non-verbal reasoning in 4-year-old children: A controlled, randomized study. Developmental Science, 14(3), 591-601. doi: 10.1111/j.1467-7687.2010.01022.x.

Brum, P. S. (2012). Treino de memória para idosos saudáveis e com comprometimento cognitivo leve: benefícios sobre parâmetros cognitivos (Dissertação de mestrado). Universidade de São Paulo, São Paulo S.P. Recuperado de http://www.teses.usp.br/teses/disponiveis/5/5142/tde-27072012152403/pt-br.php

Carvalho, F. C., Neri, A., \& Yassuda, M. S. (2010). Treino de memória episódica com ênfase em categorização para idosos sem demência e depressão. Psicologia: Reflexão e Crítica, 23(2), 317-323. doi: 10.1590/S0102-79722010000200014

Chariglione, I. P. F. (2010). A influência de diferentes tipos de treinos cognitivos na memória de idosos institucionalizados (Dissertação de mestrado). Universidade de Brasília, Brasília (DF). Recuperado de http://repositorio.unb.br/handle/10482/8164?mode=full

Chariglione, I. P. F. (2014). Intervenções cognitivas para o aprimoramento da memória em idosos com envelhecimento cognitivo normal (Tese de doutorado). Universidade de Brasília, Brasília (DF). Recuperado de http://repositorio.unb.br/handle/10482/16137

Chein, J. M., \& Morrison, A. B. (2010). Expanding the mind's workspace: Training and transfer effects with a complex working memory span task. Psychonomic Bulletin E Review, 17(2), 193-199. doi: 10.3758/PBR.17.2.193

Einstein, G. O., \& McDaniel, M. A. (2004). Memory fitness: A guide for successful aging. New Haven, CT: Yale University Press

Gamer, M., Lemon, J., Fellows, I., \& Singh, P (2012). Various Coefficients of Interrater Reliability and Agreement. Recuperado de http://CRAN.Rproject.org/package $=$ irr

Gates, N. J., Sachdev, P. S., Singh, M. A. F., \& Valenzuela, M. (2011). Cognitive and memory training in adults at risk of dementia: a systematic review. BMC. geriatrics, 11(1), 1. doi: 10.1186/1471-2318-11-55

Gelman, A., \& Hill, J. (2006). Data analysis using regression and multilevel/hierarchical models. Cambridge University Press.

Hertzog, C., Kramer, A. F., Wilson, R. S., \& Lindenberger, U. (2008). Enrichment effects on adult cognitive development: Can the functional capacity of older adults be preserved and enhanced? Psychological Science, 9(1), 1-65. doi: 10.1111/j.1539-6053.2009.01034.x

Irigaray, T. Q., Schneider, R. H., \& Gomes, I. (2004). Efeitos de um treino cognitivo na qualidade de vida e no bem-estar psicológico de idosos. Psicologia: Reflexão e Crítica, 24(4), 810-818. doi: 10.1590/S0102-79722011000400022

Irigaray, T. Q., Filho, I. G., \& Schneider, R. H (2010). Efeitos de um treino de atenção, memória e funções executivas na cognição de idosos saudáveis. Psicologia: Reflexão e Crítica, 25(1), 188-202. doi: 10.1590/S0102-79722012000100023

Jaeggi, S. M., Buschkuehl, M., Jonides, J., \& Perrig, W. J. (2008). Improving fluid intelligence with training on working memory. Proceedings of the National Academy of Sciences, 105(19), 6829-6833. doi: 10.3758/s13423-014-0699-x

Jobe, J. B., Smith, D. M., Ball, K., et al. (2001). ACTIVE: A cognitive intervention trial to promote independence in older adults. Control Clinical Trials, 22(4), 453-79. doi: 10.1001/jama.288.18.2271.

Karbach, J., \& Verhaeghen, P. (2014). Making working memory work: A meta-analysis of executive control and working memory training in older adults. Psychological Science, 25, 2027-2037. doi: 10.1177/0956797614548725 
Kelly, M. E., Loughrey, D., Lawlor, B. A., Robertson, I. H., Walsh, C., \& Brennan, S (2014). The impact of cognitive training and mental stimulation on cognitive and everyday functioning of healthy older adults: A systematic review and meta-analysis. Ageing Research Reviews, 15, 28-46. doi: 10.1016/j.arr.2014.02.004.

Klingberg, T., \& Bergman-Nutley, S. (2014). Effect of working memory training on working memory, arithmetics, and following instructions. Psychological Research (2014). doi 10.1007/s00426-014-0614-0

Lasca, V. B. (2003). Treinamento de memória no envelhecimento normal: um estudo experimental utilizando a técnica de organização (Tese de doutorado). Universidade Estadual de Campinas, São Paulo, SP. Recuperado de www.bibliotecadigital.unicamp.br/ document/?down $=$ vtls000308781

Law, L. L. F., Barnett, F., Yau, M. K., \& Gray, M. A. (2014). Effects of combined cognitive and exercise interventions on cognition in older adults with and without cognitive impairment: A systematic review. Ageing Research Review, 15, 61-75. doi: 10.1016/j.arr.2014.02.008

Lee, S., \& Shi, J. (2000). Joint Bayesian analysis of factor scores and structural parameters in the factor analysis model. Annals of the Institute of Statistical Mathematics, 52, 722-736. doi: 10.1348/000711001159546

Lee, S. Y., \& Song, X. Y. (2004) Evaluation of the bayesian and maximum likelihood approaches in analyzing structural equation models with small sample sizes. Multivariate Behavioral Research, 39(4), 653-686, doi: 10.1207/s15327906mbr3904_4

Lima-Silva et al. (2011). Treino cognitivo para idosos baseado em estratégias de categorização e cálculos semelhantes a tarefas do cotidiano. Revista Brasileira de Geriatria e Gerontologia, 14(1), 65-74. doi: 10.1590/S1809-98232011000100008

Lima-Silva, T. B. et al. (2010). Effects of cognitive training based on metamemory and mental images. Dementia and Neuropsychology, 4(2), 114-119. Recuperado de http://www.demneuropsy.com.br/imageBank/PDF/v4n2a07.pdf

Lima-Silva, T. B., \& Yassuda, M. S. (2010). Treino cognitivo e intervenção psicoeducativa para indivíduos hipertensos: Efeitos na cognição. Psicologia: Reflexão e Crítica, 25(1), 30-40. doi: 10.1590/S0102-79722012000100005

Markus, K. A., \& Borsboom, D. (2013). Frontiers of Test Validity Theory: Measurement, Causation and Meaning. Multivariate Application Series.

Martin, M., Clare, L., Altsgassen, A. M., Cameron, M. H., \& Zehnder, F. (2011). Cognition-based interventions for healthy older people and people with mild cognitive impairment. The Cochrane Library, 1, 1-51. doi: 10.1002/14651858.CD006220.pub2

Muthén, B., \& Asparouhov, T. (2012). Bayesian structural equation modeling: A more flexible representation of substantive theory. Psychological Methods, 17(3), 313.

Muthén, L. K.., \& Muthén, B. O. (2012). Mplus User's Guide (7th Edition). Los Angeles, CA: Muthén \& Muthén.

Melby-Lervag, M., \& Hulme, C. (2013). Is working memory effective? A meta-analytic review. Developmental Psychology, 49(2), 270-291. doi: $10.1037 / \mathrm{a} 0028228$

Nascimento, E. (2004). Adaptação, validação e normatização de uma amostra brasileira. Em E. Nascimento (Eds.), WAIS-III: Escala de Inteligência Wechsler para Adultos - manual para administração e avaliação (pp.161-192). São Paulo: Casa do Psicólogo

Olchik, M. R. (2008). Treino de memória: Um novo aprender no envelhecimento (Tese de doutorado). Universidade Federal do Rio Grande do Sul, Porto Alegre, RS. Recuperado de http://www.lume.ufrgs.br/handle/10183/13489

Oliveira, T. C. G., Soares, F. C., Macedo, L. D. D., Wanderley, D. L., Diniz, P., Bento-Torres, N. V. O., \& Picanço-Diniz, C. W. (2014) Beneficial effects of multisensory and cognitive stimulation on age-related cognitive decline in long-term-care institutions. Clinical Interventions in Aging, 14(9), 309-321. doi: 10.2147/CIA.S54383

Papp, K. V., Stephen, J. W., \& Peter, J. S. (2009). Immediate and delayed of cognitive interventions in healthy elderly: A review of current literature and future directions. Alzheimer's and Dementia, 5, 50-60. doi: 10.1016/j.jalz.2008.10.008

Paulo, D. L. V., \& Yassuda, M. S. (2012). Elderly individuals with diabetes: Adding cognitive training to psychoeducational intervention. Educational Gerontology, 38(4), 257-270. doi: 10.1080/03601277.2010.544575

Plumer, M., Best, N., Cowles, K., \& Vines, K. (2010). Package 'coda'. Package Reference Manual. Recuperado de http://cran.r-project.org/web/ packages/coda/coda.pdf

Press, S. J. (2009). Bayesian Factor Analysis. Em J. Press (Eds.), Subjective and objective Bayesian statistcs: Principles, models, and applications (pp 359-390), Hoboken, New Jersey, John Wiley \& Sons.

Rebok, G. W., Ball, K., Guey, L. T., Jones, R. N., Kim, H.Y., King, J. W., Marsiske, M., Morris, J. N., Tennstedt, S. L., Unverzagt, F. W., \& Willis, S. (2014). Ten-year effects of the advanced cognitive training for independent and vital elderly cognitive training trial on cognition and everyday functioning in older adult. Journal of the American Geriatrics Society, 62, 21-31. doi: 10.1111/jgs.12607

Reijnders, J., Van Heugten, C., \& Van Boxtel, M (2013). Cognitive interventions in healthy older adults and people with mild cognitive impairment: A systematic review. Ageing Research Review, 12, 263-275. doi: 10.1016/j.arr.2012.07.003

Salthouse, T. A. (2006). Mental exercise and mental aging: Evaluating the validity of the "use it or lose it" hypothesis. Perspectives on Psychological Science, 1, 68-87.

Santos, I. B. (2010). Oficinas de estimulação cognitiva em idosos analfabetos e com transtorno cognitivo leve (Dissertação de mestrado). Universidade Católica de Brasília, Brasília, DF. doi: 10.1111/j.1745-6916.2006.00005.x

Santos, M. T. (2015). Desenvolvimento de um programa de treino cognitivo para idosos (Tese de doutorado). Universidade Federal de Minas Gerais, Belo Horizonte, MG.

Schaie, K. W. (2005). Developmental influences on adult intelligence: The Seattle Longitudinal Study. New York: Oxford University Press.

Schaie, K. W., \& Willis, S. L. (1986). Can decline in adult intellectual functioning be reversed? Development Psychology, 22(2), 223-232. doi: 10.1037/0012-1649.22.2.223

Schaie, K. W., Willis, S. L., \& Caskie, G. I. L. (2004). The seattle longitudinal study: Relationship between personality and cognition. Aging, Neuropsychology and Cognition, 11, 304-324. doi: 10.1080/13825580490511134

Shubert, T., Strobach, T., \& Karbach, J. (2014). New directions in cognitive training: On methods, transfer and applications. Psychological Research, 78, 749-755. doi: 10.1007/s00426-014-0619-8.

Silva, H. S., \& Yassuda, M. S. (2009). Memory Training for older adults with low education: mental images versus categorization. Educational Gerontology, 35(10), 890-905. doi: 10.1080/03601270902782487

Soares, Z. F., Santana, E. F., \& Rabelo, D. F. (2015). Iniciação à informática associada ao treino cognitivo em idosos. Revista Ciência em Extensão, 11(3), 155-167. Recuperado de: http://ojs.unesp.br/index.php/revista_proex/article/view/1112

Strobach, T., Frensch, P. A., \& Schubert, T. (2012). Video game practice optimizes executive control skills in dual-task and task switching situations. Acta Psychologica, 140(1), 13-24. doi: 10.1016/j.actpsy.2012.02.001 
Tardif, S., \& Simard, M. (2011). Cognitive stimulation programs in healthy elderly: A review. International Journal of Alzheimer Disease, 1-14. doi: 10.4061/2011/378934

Tavares, P. N., Schimidt, J. H., \& Witter, C (2015). Efeitos de um programa de intervenção no desempenho cognitivo e sintomatologia depressiva em idosos institucionalizados. Revista Kairós Gerontologia, 18(2), 103-123. Recuperado de http://revistas.pucsp.br/index.php/ kairos/article/view/26316

Teixeira-Fabrício, A., Lima-Silva, T. B., Kissaki, P. T., Vieira, M. G., Ordonez, T. N., Oliveira, T. B., et al. (2012). Treino cognitivo em adultos maduros e idosos: Impacto de estratégias segundo faixas de escolaridade. Psico-USF, 17(1), 85-95. doi: 10.1590/S1413-82712012000100010

Urbina, S. (2007). Fundamentos da testagem psicológica. Porto Alegre: Artmed.

Valenzuela, M., \& Sachdev, P. (2009). Can cognitive exercise prevent the onset dementia? Systematic review of randomized clinical trials with longitudinal follow-up. American Journal of Geriatric Psychiatry, 17(3), 179-187. doi: 10.1097/JGP.0b013e3181953b57

Verhaeghen P., Marcoen, A., Goossens, L. (1992). Improving memory performance in the aged through mnemonic training: A metaanalytic study. Psychology Aging, 72, 242-251.

Willis, S. L., \& Nesselroade, C. S. (1990). Long term effects of fluid ability training in old-old age. Developmental Psychology, 26, 905-910. Recuperado de http://psycnet.apa.org/index.cfm?fa=buy.option ToBuy\&id=1991-07074-001

Willis, S. L., Tennstedt, S. L., Marsiske, M., Ball, K., Elias, J., Koepke, K. M., ... \& Wright, E. (2006). Long-term effects of cognitive training on everyday functional outcomes in older adults. Jama, 296(23), 2805-2814.

Wood, G. M. O. (2000). Efeitos do nível de auto-eficácia cognitiva percebida e de programas de treinamento cognitivo sobre a capacidade de memória de trabalho de indivíduos idosos (Dissertação de mestrado). Universidade Federal de Minas Gerais, Belo Horizonte, MG.

Yassuda, M. S., Batistoni, S. S. T., Fortes, A. G., \& Neri, A. L. (2006). Treino de memória no idoso saudável: Benefícios e mecanismos. Psicologia: Reflexão e Crítica, 19(3), 470-481. doi: 10.1590/S0102-79722006000300016

Zimmermann, N., Netto, T. M., Amodeo, M. T., Ska, B., Fonseca, R. P. (2014) Working memory training and poetry-based stimulation programs: Are the differences in cognitive outcomes in healthy older adults? NeuroRehabilitation, 35(1), 159-170. doi: http://dx.doi. org/10.3233/NRE-141104

\section{Sobre os autores}

Mariana Teles Santos Golino é psicóloga, Doutora em Psicologia do Desenvolvimento Humano pela Universidade Federal de Minas Gerais, docente no Instituto Multidisciplinar em Saúde, Universidade Federal da Bahia.

Patrícia Waltz Schelini é psicóloga, Mestre e Doutora em Psicologia pela Pontifícia Universidade Católica de Campinas, Pós- Doutora pela Universidade do Minho e Professora dos cursos de graduação e pós-graduação em Psicologia da Universidade Federal de São Carlos.

Hudson Fernandes Golino é psicólogo, Doutor em Neurociências pela Universidade Federal de Minas Gerais, Professor Assistente de Métodos Quantitativos no Departamento de Psicologia, Universidade da Virgínia.

Bruna Luísa de Souza Pereira é psicóloga, Mestranda em Psicologia pela Universidade Federal de Minas Gerais.

Lucas Matias Félix é psicólogo, Mestrando em Psicologia pela Universidade Federal de Minas Gerais. 